九州大学学術情報リポジトリ

Kyushu University Institutional Repository

Pseudo-Relativistic Vector Analysis of Animal Growth in Four-Dimensional Space-Time : Preliminary Report with Problem of Dimensional Inconsistency between Space and Time

Shimojo, Masataka

Laboratory of Regulation in Metabolism and Behavior, Division of Animal and Marine Bioresource

Sciences, Department of Bioresource Sciences, Faculty of Agriculture, Kyushu University

Nakano, Yutaka

University Farm, Faculty of Agriculture, Kyushu University

https://doi.org/10.5109/1434384

出版情報 : 九州大学大学院農学研究院紀要. 59 (1)，pp.91-92，2014-02-28. Faculty of Agriculture， Kyushu University

バージョン :

権利関係: 


\title{
Pseudo-Relativistic Vector Analysis of Animal Growth in Four-Dimensional Space-Time - Preliminary Report with Problem of Dimensional Inconsistency between Space and Time -
}

\author{
Masataka SHIMOJO* and Yutaka NAKANO ${ }^{1}$ \\ Laboratory of Regulation in Metabolism and Behavior, Division of Animal and Marine Bioresource Sciences, \\ Department of Bioresource Sciences, Faculty of Agriculture, \\ Kyushu University, Fukuoka 812-8581, Japan \\ (Received October 31, 2013 and accepted November 11, 2013)
}

\begin{abstract}
This study investigated the space-time relationship in the growth analysis of an individual animal by introducing a pseudo-relativistic vector, under the problem of dimensional inconsistency between space and time. Space and time were treated equally and growth vector inclining to space-time axes was introduced. The growth vector $(\boldsymbol{G})$ of the animal was described using a pseudo-relativistic vector in four-dimensional space-time, $\boldsymbol{G}=(x, y, z, t)$. The magnitude of $\boldsymbol{G}(G m)$ was regarded as an index of the four-dimensional growth of the animal. The results obtained were as follows. (i) If $\boldsymbol{G}$ inclined greatly to $z$-axis compared with the other three axes $(x, y, t)$, then this was a result of the fact that the animal became taller by the efficient utilization of the height direction in space. (ii) If $\boldsymbol{G}$ inclined greatly to $t$-axis compared with space axes $(x, y, z)$, then this was a result of the fact that the animal showed a smaller growth because of the inefficient utilization of time. (iii) An invariant connecting geometric and analytic representations of growth was proposed to make an inclusive comparison of various cases of animal growth. It was suggested that space and time were related in a pseudo-relativistic vector analysis of animal growth, under geometric and analytic representations.
\end{abstract}

Key words: Animal growth, four dimension, pseudo-relativity, space-time, vector analysis

\section{INTRODUCTION}

The animal production is influenced by how efficiently the animal utilizes space and time for its growth. The animal growth is usually analyzed along one-dimensional time axis or along three-dimensional space axes. This is a traditional growth analysis method that has a long history, where space and time is treated separately. If four-dimensional space-time is applied, then this may give information on which of space and time is efficiently utilized for the animal production. Shimojo et al. (2009) proposed a quasi-four-dimensional growth model to analyze the weight-space-time relationship in an individual plant or animal, but this issue is very complicated.

The present study investigated the space-time relationship in the growth analysis of an individual animal by introducing a pseudo-relativistic vector, under the problem of dimensional inconsistency between space and time.

\section{PSEUDO-RELATIVISTIC VECTOR ANALYSIS OF ANIMAL GROWTH}

\section{Description of the growth of an individual animal using a pseudo-relativistic vector}

The growth vector $(\boldsymbol{G})$ of an individual animal is hypothetically described using a pseudo-relativistic vec-

${ }^{1}$ University Farm, Faculty of Agriculture, Kyushu University, Kasuya, Fukuoka 811-2307

* Corresponding Author (E-mail: mshimojo@agr.kyushu-u.ac.jp) Part of this study was conducted at Agri-Bio Research Laboratory on Ito Campus, Kyushu University, Motooka, Fukuoka 819-0395 tor in four-dimensional space-time. Thus,

$$
\boldsymbol{G}=(x, y, z, t),
$$

where $x=$ width $(\mathrm{cm}), y=\operatorname{depth}(\mathrm{cm}), z=\operatorname{height}(\mathrm{cm})$, $t=$ time (day).

The above units are one example. There is a problem of dimensional inconsistency between space and time in $\boldsymbol{G}$. However, treating space and time equally and introducing $\boldsymbol{G}$ that inclines to space-time axes might be related to pseudo-relativistic characteristics.

\section{Geometric representation of four-dimensional growth}

The magnitude of $\boldsymbol{G}(\mathrm{Gm})$ is regarded hypothetically as an index of the four-dimensional growth of an individual animal. Thus,

$$
\begin{aligned}
& G m=\sqrt{x^{2}+y^{2}+z^{2}+t^{2}}, \\
& G m \cdot \cos \alpha=x, \\
& G m \cdot \cos \beta=y, \\
& G m \cdot \cos \gamma=z, \\
& G m \cdot \cos \delta=t,
\end{aligned}
$$

where $\alpha=$ the angle between $\boldsymbol{G}$ and $x$-axis, $\beta=$ the angle between $\boldsymbol{G}$ and $y$-axis, $\gamma=$ the angle between $\boldsymbol{G}$ and $z$-axis, $\delta=$ the angle between $\boldsymbol{G}$ and $t$-axis.

It is possible to describe the space axes, but it is impossible to describe the time axis whose direction is invisible. Thus, only the angle between $\boldsymbol{G}$ and $t$-axis is hypothesized.

Two examples of the geometric representation of the four-dimensional growth are as follows. (i) If $\boldsymbol{G}$ inclines 
greatly to $z$-axis compared with the other three axes ( $x$, $y, t)$, then this is a result of the fact that the animal becomes taller by the efficient utilization of the height direction in space. (ii) If $\boldsymbol{G}$ inclines greatly to $t$-axis compared with space axes $(x, y, z)$, then this is a result of the fact that the animal shows a smaller growth because of the inefficient utilization of time. These interpretations are based on the following. If $x>y>z>t$, then $\alpha$ $<\beta<\gamma<\delta$; namely $\cos \alpha>\cos \beta>\cos \gamma>\cos \delta$.

\section{Analytic representation of four-dimensional growth}

We will hypothesize that the rates of growth along space-time axes are given by the partial differentiation of $\mathrm{Gm}$. Thus,

$$
\begin{aligned}
& \frac{\partial G m}{\partial x}=\frac{x}{\sqrt{x^{2}+y^{2}+z^{2}+t^{2}}}, \\
& \frac{\partial G m}{\partial y}=\frac{y}{\sqrt{x^{2}+y^{2}+z^{2}+t^{2}}}, \\
& \frac{\partial G m}{\partial z}=\frac{z}{\sqrt{x^{2}+y^{2}+z^{2}+t^{2}}}, \\
& \frac{\partial G m}{\partial t}=\frac{t}{\sqrt{x^{2}+y^{2}+z^{2}+t^{2}}} .
\end{aligned}
$$

The comparison with the preceding section suggests that the analytic representation using the partial differentiation of $G m$ is the same as the geometric representation using the cosine function of $\mathrm{Gm}$.

\section{Geometric and analytic invariant}

There is an invariant that is conserved by the geometric and analytic representations where every term is squared. Thus,

$$
\begin{aligned}
& \left(\frac{\partial G m}{\partial x}\right)^{2}+\left(\frac{\partial G m}{\partial y}\right)^{2}+\left(\frac{\partial G m}{\partial z}\right)^{2}+\left(\frac{\partial G m}{\partial t}\right)^{2} \\
& =\cos ^{2} \alpha+\cos ^{2} \beta+\cos ^{2} \gamma+\cos ^{2} \delta \\
& =1 .
\end{aligned}
$$

This invariant suggests that each of the four dimensions is related to the other three. Expression (4) might be applied to an inclusive comparison of various cases of animal growth.

\section{Growth force (the product of growth $(\mathrm{Gm})$ and growth acceleration)}

We will hypothesize that the acceleration of the growth along $x$-axis is given by

$$
\begin{aligned}
& \frac{\partial^{2} G m}{\partial x^{2}} \\
= & \frac{1}{\sqrt{x^{2}+y^{2}+z^{2}+t^{2}}} \cdot \frac{y^{2}+z^{2}+t^{2}}{x^{2}+y^{2}+z^{2}+t^{2}}
\end{aligned}
$$

$$
=\frac{1}{G m} \cdot\left(\left(\frac{\partial G m}{\partial y}\right)^{2}+\left(\frac{\partial G m}{\partial z}\right)^{2}+\left(\frac{\partial G m}{\partial t}\right)^{2}\right) .
$$

This leads to the growth force (the left-hand side of expression (5-1)) along $x$-axis,

$$
G m\left(\frac{\partial^{2} G m}{\partial x^{2}}\right)=\left(\frac{\partial G m}{\partial y}\right)^{2}+\left(\frac{\partial G m}{\partial z}\right)^{2}+\left(\frac{\partial G m}{\partial t}\right)^{2},
$$

and likewise,

$$
\begin{aligned}
& G m\left(\frac{\partial^{2} G m}{\partial y^{2}}\right)=\left(\frac{\partial G m}{\partial z}\right)^{2}+\left(\frac{\partial G m}{\partial t}\right)^{2}+\left(\frac{\partial G m}{\partial x}\right)^{2}, \\
& G m\left(\frac{\partial^{2} G m}{\partial z^{2}}\right)=\left(\frac{\partial G m}{\partial t}\right)^{2}+\left(\frac{\partial G m}{\partial x}\right)^{2}+\left(\frac{\partial G m}{\partial y}\right)^{2}, \\
& G m\left(\frac{\partial^{2} G m}{\partial t^{2}}\right)=\left(\frac{\partial G m}{\partial x}\right)^{2}+\left(\frac{\partial G m}{\partial y}\right)^{2}+\left(\frac{\partial G m}{\partial z}\right)^{2} .
\end{aligned}
$$

Each growth force is related to the sum of the other three growth rates squared, which seems to be a space-time continuum. This might be related to a pseudo-relativistic characteristic of the four-dimensional growth of an individual animal.

The sum of expressions (5-1) (5-4) gives

$$
\begin{aligned}
& G m\left(\frac{\partial^{2} G m}{\partial x^{2}}+\frac{\partial^{2} G m}{\partial y^{2}}+\frac{\partial^{2} G m}{\partial z^{2}}+\frac{\partial^{2} G m}{\partial t^{2}}\right) \\
& =3\left(\left(\frac{\partial G m}{\partial x}\right)^{2}+\left(\frac{\partial G m}{\partial y}\right)^{2}+\left(\frac{\partial G m}{\partial z}\right)^{2}+\left(\frac{\partial G m}{\partial t}\right)^{2}\right), \\
& =3\left(\cos ^{2} \alpha+\cos ^{2} \beta+\cos ^{2} \gamma+\cos ^{2} \delta\right) \\
& =\sin ^{2} \alpha+\sin ^{2} \beta+\sin ^{2} \gamma+\sin ^{2} \delta \\
& =3
\end{aligned}
$$

Expressions (6) (9) suggest a hypothesis that growth force is a fundamental concept for the analytic and geometric representations of animal growth in fourdimensional space-time.

\section{Conclusions}

This study suggests that space and time are related in a pseudo-relativistic vector analysis of animal growth, under geometric and analytic representations.

\section{REFERENCES}

Shimojo, M., Y. Asano, R. Ishiwaka, Y. Nakano, M. Tobisa, N. Ohba, M. Eguchi and Y. Masuda 2009 Introducing viewpoints of mechanics into basic growth analysis - (IX) Hypothetic quasifour-dimensional growth mechanics -. J. Fac. Agr., Kyushu Univ., 54: 137-139 